\title{
Current and future biosimilars: potential practical applications in rheumatology
}

This article was published in the following Dove Press journal:

Biosimilars

31 July 2013

Number of times this article has been viewed

\section{Ghaith Noaiseh \\ Larry Moreland}

Division of Rheumatology and Clinical Immunology, University of Pittsburgh Medical Center, Pittsburgh, PA, USA
Correspondence: Ghaith Noaiseh Division of Rheumatology and Clinical Immunology, University of Pittsburgh Medical Center, 3500 Terrace Street BST-S 7I8, Pittsburgh, PA I526I, USA Tel +l 4I26489782

$\mathrm{Fax}+$ I 4123838753

Email noaisehg@upmc.edu

\begin{abstract}
The use of biologics in the field of rheumatology has dramatically changed the way we treat rheumatic diseases. As the patent-expiration dates for many tumor necrosis-factor inhibitors and other biological agents are approaching, many large pharmaceutical companies are developing and testing their own versions of these agents; this is due to the biologics' huge revenue potential. The potential cost saving is a major incentive for their development. Producing a biosimilar is not an easy task, as minor changes in the production process can have profound immunological and clinical consequences. The European Medicines Agency (EMA) has led the efforts in issuing guidelines to streamline the approval process for applicants interested in developing biosimilars. The US Food and Drug Administration (FDA) has followed the EMA track and has guidelines in place, but the process varies in different countries. The approval process is far more complex than the one used for the approval of small-molecule generic products. Biosimilars should be developed according to the strict rules set forth by the EMA and FDA; other intended copies are available for clinical use in different parts of the world, but should not be considered biosimilars, as they do not fulfill the stringent definition criteria. Biosimilars will soon be in the market, and their use in rheumatic diseases will likely change our treatment approach. Rheumatologists and other health-care professionals will soon be faced with many questions and will have to be familiarized with the concept and the points of debate.
\end{abstract}

Keywords: biosimilar, rheumatology, biologic, generic, TNF inhibitor, rituximab

\section{Introduction}

The introduction of targeted biologic therapies has revolutionized the treatment of many rheumatic diseases, including rheumatoid arthritis (RA), psoriatic arthritis, and ankylosing spondylitis (AS). These agents exhibit their effect by affecting inflammatory cells and cytokines that play pivotal roles in inflammation, decreasing disease activity and reducing structural damage to joints. ${ }^{1}$ Their use in the management of rheumatic diseases has significantly improved patient outcomes. ${ }^{2}$ Furthermore, compared with traditional RA therapies, some authors advocate using tumor necrosis-factor inhibitors (TNFis) and other biologics more often than they are currently used, possibly to include patients with clinically moderate disease. ${ }^{3}$

Around US\$130 billion was spent on biologics in 2009 worldwide. ${ }^{4}$ Total annual sales of biologics for rheumatic disorders are estimated to be $\$ 30$ billion. ${ }^{5}$ This amount is approximated to be $\$ 10,000-\$ 30,000$ per patient per year. ${ }^{5}$ In 2010 , rituximab sales reached $\$ 6.6$ billion, making this agent the largest revenue-making biologic in the market. ${ }^{4}$ More recently, in 2012, worldwide sales of etanercept, adalimumab, and infliximab, the top selling TNFi agents, reached $\$ 20$ billion. $^{6}$ The high cost of biologics 
raises concerns about the financial burden to patients and health-care payers, particularly as new products become available and their use for the treatment of autoimmune diseases continues to expand. ${ }^{4}$

The elevated acquisition costs of TNFis may be a barrier that prevents patients with RA from utilizing these agents. ${ }^{7}$ In addition, there is a humanistic burden due to restricted access caused by budget constraints in many countries around the world. ${ }^{5}$ Many pharmaceutical companies are now developing their own versions of these agents, since many of these reference (or originator) products are approaching their patent-expiration dates. ${ }^{8}$ The development of biosimilars is understandably driven by the revenue potential. According to one report, 74\% of Thousand Oaks, CA-based Amgen's 2010 revenue (estimated at $\$ 11$ billion) and $57 \%$ of Bostonbased Genzyme's 2010 revenue (estimated at $\$ 2.3$ billion) would be exposed through patent expiration by $2015 .{ }^{9}$

The advantage of biosimilars when compared to the originator products is the potential cost saving. This has been shown to be real in the erythropoietin market: the projected saving in Germany by 2020, for example, will be around $€ 8$ billion, which implies the potential for better patient access. ${ }^{3}$ The US Congressional Budget Office has estimated that availability of biosimilars could save the nation up to $\$ 25$ billion over the next 10 years. ${ }^{9}$ Availability of biosimilars in the US is anticipated not only to enhance competition and create better patient access to biotechnology products but also to lower their cost. ${ }^{9}$ It is currently difficult to predict cost savings for biosimilar monoclonal antibodies (mAbs) and receptor fusion proteins (-cepts) in highly regulated markets (as in the US and EU). ${ }^{1}$ The order of magnitude of cost saving in producing biosimilars may not be as robust as with the case of generic medications, due to elevated manufacturing costs and the need for extensive clinical and nonclinical studies. ${ }^{1,10,11}$

Given the increased attention to the topic, more relevant articles have started to appear. As of April 15, 2013, combining search words of "rheumatic" or "rheumatology" with "biosimilar," "biosimilars," or "follow-on biologics" on the PubMed website yielded ten articles. The first article was published in 2011. This includes landmark articles with comprehensive reviews of the role of these agents in the management of rheumatic diseases. ${ }^{5}$ In 2010 , a seven-question survey conducted in Brazil ${ }^{12}$ evaluated rheumatologists' familiarity with biosimilars. A total of 200 practicing rheumatologists and rheumatology trainees were approached during a national rheumatology conference. The response rate was $95 \% ; 18 \%$ of respondents were trainees.
Approximately one-third of the group did not know what a biosimilar was; this was felt to be a reasonable percentage by the investigator, since the subject was new. ${ }^{12}$ To our knowledge, we are not aware of similar studies to address rheumatologists' acquaintance with this field in other parts of the world, but it may be reasonable to assume it will not be a lot different. This effort highlights the importance of educating rheumatologists across the world about this soonto-be practice-changing field.

\section{Definitions}

The term "biosimilar" has created a great deal of confusion and been inconsistently used. ${ }^{13} \mathrm{~A}$ biosimilar product is defined in the US as one that is "highly similar to the reference product notwithstanding minor differences in clinically inactive components" and for which there are "no clinically meaningful differences between the biological product and the reference product in terms of safety, purity and potency."14 Europeans define a biosimilar as a "copy version of an already authorized biological medicinal product with demonstrated similarity in physicochemical characteristics, efficacy and safety, based on a comprehensive comparability exercise." 14 The concept is subject to the strict definition of the authorizing agencies in a tightly regulated market (the European Medicines Agency [EMA] and the US Food and Drug Administration [FDA]). In other words, for a medicine to be called a biosimilar, strict rules, not available in other intended copies of the reference product, must apply.

Probably the best effort to date that outlined the differences in terminology and their clinical implications pertinent to this topic was made by Weise et al: ${ }^{13}$

- The term "biologics" refers to a class of medications produced by living cells using recombinant DNA technology. ${ }^{15}$ The recombinant DNA is introduced into the cells, which decode the DNA and produce complex proteins that can be purified and used for therapeutic purposes. ${ }^{10}$

- The "originator" biologic (or reference product) is the authorized, patented product of which other manufacturers are attempting to create a copy.

- A generic is an exact copy of a small-molecule product that is both therapeutically and structurally identical to the reference product. ${ }^{5}$

- A biosimilar (Europe, US), follow-on biological/biologic (the former term in the US), subsequent-entry biologicals/ biologics (Canada), or similar biopharmaceutical products (World Health Organization [WHO]), is a copy version of an authorized biologic that fulfills the stringent 
criteria outlined by the FDA and EMA. Only minimal, clinically irrelevant structural differences are allowed.

- A "biobetter" (second-generation or next-generation) biological/biologic is a biologic that has been structurally and/or functionally altered to achieve an improved or different clinical performance. ${ }^{13}$ One example is developing a humanized monoclonal antibody (second generation) from a chimeric monoclonal antibody (the first generation) targeting the same antigen. These are intended to improve performance of the first-generation product while preserving the mechanism of action, but are not considered biosimilars. ${ }^{16}$

- A "me-too" (or noninnovator) biological/biologic is a biologic product developed with the same target antigen, but comparability has not been demonstrated; clinical comparative studies with the reference product may or may not have been conducted. 5,13

It has been advised that the term "biogeneric" be avoided, as it is arguably incorrect from the scientific point of view. ${ }^{13}$ The notion is that the term "generic" indicates an identical replication of the original product, and unlike small-molecule generic drugs, a biosimilar is never identical to its reference product. ${ }^{16}$ Furthermore, the term "generic" refers to a different regulatory pathway, which is not scientifically sufficient for the development of a biosimilar per se.

In summary, any copy version of a therapeutic protein, not developed and assessed in concert with the scientific principles of a strictly comparative development program against a reference product, should not be referred to as a biosimilar. ${ }^{13}$ This does not imply that the other products are of lower quality, efficacy, or safety; they simply do not qualify as biosimilars according to the definition in the EU and US. In fact, neither inferiority nor superiority would be allowed. ${ }^{14,17}$ A different and unique terminology is paramount to enable a clear distinction between the different products.

\section{Structural complexity of biologics}

As mentioned before, biologics are proteins produced by recombinant DNA technology. ${ }^{15}$ Typical biologics include proteins that are intended to replace deficient hormones (as in gonadotropin-releasing hormone) or growth factors that enhance the body response (as in erythropoietin); these exhibit well-characterized structural features that can be replicated reliably using recombinant techniques. ${ }^{8}$

Other biologics include mAbs and -cepts that bind to target receptors or inflammatory cells, interfering with the signaling pathway, ${ }^{10}$ which subsequently leads to the desired clinical effect. These products are highly complex molecules with high molecular weight - around 150,000 $\mathrm{Da}$ for an $\mathrm{mAb}^{18}$ - and their synthesis is far from straightforward, as the structural complexity is not only influenced by the amino acid sequence but also by many other factors, such as the way these proteins are folded and subsequent chemical reactions such as methylation, glycosylation, and deamidation (these are referred to as posttranslational modifications), which can affect higher-order structures and are considered essential to their intended effect. Manufacturing of mAbs requires highly controlled settings ${ }^{19}$ and a special storage environment to maintain stability. ${ }^{20}$ Even subtle changes in the conformational structure of a protein (whether intentional or inadvertent modification) may lead to altered function, insolubility, or immunogenicity, leading to a change in efficacy and safety. ${ }^{11,18}$

There are many examples in the literature on how a minimal change in the synthesis or storage conditions can significantly alter the immunogenicity of the product, leading to unwanted side effects. ${ }^{5}$ One example is the development of pure red cell aplasia in a few patients who used recombinant erythropoietin, where a change in the protein stabilizer from albumin to polysorbate 80 by the manufacturer led to crossreacting antibodies to endogenous erythropoietin. ${ }^{15}$

\section{Microheterogeneity and comparability-exercise concepts}

Pharmaceutical companies are constantly trying to improve the production conditions for their licensed products through ongoing manufacturing changes. There is also an inherent variability in the biological systems used in manufacturing attributed to technical and scientific limitations. ${ }^{21}$ For these reasons, the resulting biological product is destined to display some degree of variability over time; this phenomenon is referred to as microheterogeneity. ${ }^{13}$ It is a feature of batchto-batch variability for any biological agent, ${ }^{22}$ and is not only considered "normal" and part of the "life cycle" of the product but is also welcome, as this often means improvement. For example, some manufacturing changes to the production of interferon beta have resulted in decreased immunogenicity. ${ }^{16}$ All the approved mAbs and -cepts have undergone changes in the manufacturing process following the initial approval. ${ }^{21}$ It must be borne in mind that these "developmental" changes in manufacturing have been supported by appropriate data and approved by the authorizing agencies. ${ }^{21}$ This is referred to as a comparability exercise.

\section{Manufacturing biosimilars}

Attempting to synthesize a "highly similar" copy of the originator product is a much more complex task compared 
to reproducing a small and more stable chemical compound, such as a generic. In the case of biosimilars, the final product is influenced by many factors, such as the cell type used to produce the protein, culture conditions, posttranslational modifications, purification methods, stabilization, storage conditions, and packaging. ${ }^{10}$ Due to unavailability of the proprietary data to the developers of biosimilars, they must create their own manufacturing process, like using their own purification and fermentation methods. For this reason, the quality attributes of the biosimilar will not be identical to the originator product. ${ }^{13}$ It is safe to say that it is almost impossible to create an exact copy of the originator product. ${ }^{5,10,13}$ Thus, the key question for biosimilars is whether the difference is clinically relevant, not if it exists. ${ }^{5}$

What, then, are the fundamental features that must be retained when creating a biosimilar? ${ }^{5}$

- Primary amino acid sequence

- Potency

- Route of administration, although the administration device may be different.

On the other hand, higher-order structures, posttranslational modifications, and other potential variants must be as similar as possible to the originator product, and adequate analytical assessment must be conducted to demonstrate that any difference will not affect the clinical efficacy, safety, or immunogenicity.

Finally, the notion that "minimal changes in the manufacturing process of a biosimilar can lead to a major impact on its function" quoted in many reviews of this topic, has been misinterpreted and given unwanted implications, this was highlighted by Schneider ${ }^{21}$ in a recent editorial: "This has often had the connotation to implicitly assume that biosimilars therefore may have an undetected 'inferior' quality compared with the established originators, or that at least there is more uncertainty around them." The author points out that "The 'art' for a biosimilar is to demonstrate that the biosimilar is as close as possible to its reference product in all relevant functional and structural aspects, again within current technical and scientific possibilities and its inherent variability"

\section{Legislation} In the US

In 1984, the US Congress approved the Drug Price Competition and Patent Term Restoration Act, which established an abbreviated new-drug application pathway for the development of generic products. ${ }^{10}$ Prior to 2010, the FDA had had limited authority in approving biosimilars, resulting in delays of the development of these agents in the US compared to Europe. This changed with the passage of the Patient Protection and Affordable Care Act (the health-care reform) in March 2010. ${ }^{23}$ The law outlines an abbreviated approval pathway for biological products that are demonstrated to be highly similar to or interchangeable with a licensed biological product; it is known as the Biologics Price Competition and Innovation Act (BPCIA). ${ }^{16}$

The law gives 12 years of exclusivity to the manufacturer of an originator biologic, during which biosimilars may not be approved. ${ }^{16}$ To encourage the development of biosimilars, the BPCIA grants 1 year of exclusive marketing rights to the first biosimilar that is approved as being "interchangeable" with a reference product. ${ }^{16}$ In February 2012, The FDA issued three draft guidance documents on biosimilar product development to assist industry in developing such products in the US. They are not yet aimed at becoming "enforceable guidelines," but rather suggestions to manufacturers. ${ }^{24}$

\section{In Europe}

In 2005, the Committee for Medicinal Products for Human Use (CHMP) of the EMA, which is responsible for the scientific assessment of human medicines authorized and marketed in Europe, issued the first regulatory guidance outlining the data required to apply for a licensing of a biosimilar agent. ${ }^{1}$ In May 2012, guidance for the approval of biosimilar agents containing mAbs was issued, and came into effect in December 1, 2012. ${ }^{25}$

\section{In countries outside the EU and the US}

The regulatory framework for biosimilars varies widely. ${ }^{26}$ Some countries adopted the EMA guidelines; ${ }^{20}$ others, as with Canada, issued their own guidelines, which were mainly influenced by FDA guidelines, although there are some differences. ${ }^{1}$ In some countries in Latin America, agents were even approved before adequate data were available, as in the case of Etanar (an intended biosimilar to etanercept), which was approved in Colombia for the management of RA. Some countries based their approval pathways on the FDA or WHO, but it was ultimately left to the local regulatory authorities to decide about the type of studies required for approval. ${ }^{26}$

\section{Approval procedures}

In general, the approval of a generic product requires a demonstration of bioequivalence, or pharmacokinetic equivalence. This is usually accomplished by comparing the candidate agent to the reference product in healthy volunteers, and includes a comparison of absorption, rate of 
absorption, and peak concentration. ${ }^{20}$ Generic equivalents of many drugs proved to be relatively straightforward to produce and resulted in enormous savings. ${ }^{10}$

For the approval of biosimilars, it is very important to realize that the process relies in part on the extensive knowledge available for the originator products. ${ }^{13}$ It is not scientifically beneficial and probably even unethical to repeat the entire development program for the reference product. ${ }^{11}$ The key point for the development program is to demonstrate similarity rather than patient benefit, as this has already been achieved for the reference product. ${ }^{13}$

EMA and FDA guidelines share the same broad requirements for biosimilar approval: the biosimilar must have the same mechanism of action, route of administration, dosage, and potency as the originator product, and can only be approved for the same indications for which the originator product was licensed. ${ }^{16}$ Analytical and animal studies along with clinical trials, done on subjects of the same disease for which the originator product was licensed, are needed. ${ }^{16}$ Both agencies require randomized clinical trials that have sufficient sample sizes to establish clinical equivalence; there is also emphasis on long-term efficacy and safety assessed through postmarketing surveillance, similar to the case for originator biologics. ${ }^{5}$ This is mainly due to the unpredictable immunogenicity of the biosimilars and its potential of altering the pharmacokinetics and pharmacodynamics of the product. ${ }^{20}$

As previously discussed with the comparability-exercise concept, manufacturing changes aiming at improving the quality of the product are allowed to occur to the originator product without the need to undertake a completely new product-development program. ${ }^{23}$ Comparability exercises serve as a "bridge" between the authorized originator product and the approval of its improved copy, using analytical data alone in most situations. ${ }^{23}$ In a few cases where analytical analyses are not sufficient, preclinical and/or clinical data are required. This scientific approach for approval is identical to the approval mechanism for biosimilars, as both attempt to avoid undertaking an entirely new product-development program. Nevertheless, since the developers of biosimilars do not have access to all the manufacturing data of the originator, some level of structural differences is expected, and thus safety and efficacy cannot be sufficiently predicted without clinical studies.

Although the terms "abbreviated," "abridged," or "accelerated" have been used to describe the approval process of biosimilars, this should not be perceived as implying a shorter or less robust appraisal process. For example, the time from the start of the regulatory reviewing process to approval by the EMA was not shorter for biosimilars authorized in Europe compared with mAbs or -cepts approved in rheumatology. ${ }^{21}$

\section{Debatable concepts Extrapolation of indications}

If an originator drug is approved for diseases $\mathrm{A}$ and $\mathrm{B}$ and a candidate drug has shown biosimilarity in disease $\mathrm{A}$, could it be extrapolated that the candidate drug may be used to treat disease B? Both the EMA and FDA advocate extrapolation, 5 particularly when the mechanism of action of the biologic is the same. However, if the mechanism of action is considered different due to a different disease biology (for example, rituximab for RA and non-Hodgkin's lymphoma) or when the mechanism of action of the drug is not understood, then separate clinical trials may be necessary. ${ }^{5}$

\section{Interchangeability}

With the inclusion of interchangeability to the definition of biosimilars, the bar is raised quite high. Pharmacists can then substitute a biosimilar for the originator product without consulting with the treating rheumatologist. This implies that an applicant must provide sufficient data to demonstrate biosimilarity first, and then show that a biosimilar product may not exhibit any clinically relevant immunogenicity that can affect its safety or efficacy when alternating with the originator. ${ }^{27}$

Given issues related to immunogenicity, interchangeability is probably one of the biggest challenges to tackle in the biosimilar-approval process. ${ }^{10}$ This major technical hurdle has been addressed by some regulatory agencies, like Health Canada, which does not support interchangeability of biosimilars. ${ }^{20}$

Many regulatory agencies agree that each biologic product must have a unique product name under the International Nonproprietary Names Program of the WHO. A unique name will assist in the accurate prescribing and dispensing of biosimilars, and will support governments' efforts to monitor adverse events closely. Without distinct names, patients, physicians, and pharmacists could become confused, leading to inadvertent product substitution. ${ }^{20}$

\section{Available biosimilars for management in rheumatic diseases worldwide}

Following the issuance of regulatory guidance by the EMA, a number of biosimilar agents have been licensed in Europe. ${ }^{4}$ As of 2011, 14 products had been approved (erythropoietin, filgrastim, and somatotropin biosimilars). ${ }^{28}$ 
Currently, there are no EMA- or FDA-approved biosimilar $\mathrm{mAbs}$ or -cepts for treatment of rheumatic diseases. However, randomized controlled trials are being conducted on many candidate products, and a few have been completed. ${ }^{1}$ For example, Pfizer (USA), Teva Pharmaceuticals (Israel), Samsung (South Korea), Sandoz (Switzerland), Celltrion (South Korea), Boehringer (Germany), and Merck (USA) are performing, or have performed, phase I and II trials on rituximab biosimilars. ${ }^{5}$

Perhaps the closest candidate product to getting approved in a highly regulated market is CT-P13, a candidate biosimilar to infliximab produced by the South Korean company Celltrion. CT-P13 was approved by the Korean Food and Drug Administration on July 20, 2012 for the management of RA and AS under the brand name Remsima. ${ }^{29}$ The approval was based on two pivotal studies: the first, ${ }^{30}$ was a phase I study involving 250 patients with $\mathrm{AS}$, and the second ${ }^{31}$ involved 606 patients with RA. Celltrion filed CT-P13 in Europe, and was accepted for review by the EMA in March 2012. It was the first biosimilar monoclonal antibody to be filed for approval in Europe. The two successful trials will likely help Celltrion to become the pharmaceutical company producing the world's first biosimilar agent to be marketed in the EU or the US. ${ }^{32}$ The patent for infliximab is expected to expire by 2014 in Europe and Japan.

Intended replicas of originators are already in use for management of RA in many countries, such as Chile, Bolivia, and the People's Republic of China. ${ }^{5}$ As discussed before, these should not be considered biosimilars despite their local approval, as they do not fulfill the stringent FDA and EMA criteria.

An intended copy of etanercept, manufactured by CP Guojian Pharmaceutical in the People's Republic of China, has already been marketed in the People's Republic of China as Yisaipu since 2005, and in Colombia as Etanar. ${ }^{33}$ Reditux, an intended copy of rituximab produced by Dr Reddy's Laboratories in India, has become available in some countries of Latin America, including Peru, Ecuador, Chile, Bolivia, and Venezuela, ${ }^{34}$ despite the paucity of data regarding its efficacy. $^{20}$

\section{Future studies to demonstrate similarity}

Current EMA guidelines set by its $\mathrm{CHMP}^{25}$ call for a "smart" approach in choosing patient populations for comparative clinical trials. Efficient study models are suggested to tackle the high cost and complexity by running simpler and smaller trials. ${ }^{34}$ The notion is to:
- choose a sensitive experimental human model whereby the clinical impact of the originator product is large; thus, by comparing two large impacts, small differences can be easily appreciated

- use a homogeneous cohort of patients that eliminates the intersubject variability and allows variability to be more noticeable between the candidate biosimilars and the originator product

- choose younger patient populations to eliminate the noise from comorbid conditions in older patients, ${ }^{34}$ this can lead to a smaller sample size.

There may be strategies that allow a "fingerprint"-like identification of highly similar patterns in two different products, which might be used to reduce the scope and extent of the currently required clinical studies. ${ }^{11}$ Other purely analytical studies might obviate the need for clinical work altogether, although this seems extremely unlikely, given the current state of technology and experience gained so far. $^{34}$

\section{The future}

Authorized biosimilars should be "as good, safe and efficacious as originator biologics." ${ }^{21}$ Their role in the management of rheumatic disease, however, will be based on the confidence gained by the treating rheumatologist. ${ }^{5}$ It is paramount that manufacturing companies strictly adhere to the guidelines set forth by the EMA and FDA to meet the high-quality standards of biosimilarity. Rheumatologists in the US, EU, and the rest of the world will, sooner or later, be utilizing a wide range of alternative options to many patented originator biologics. It is likely that the implementation of biosimilars in the management of different rheumatic diseases will change the treatment algorithms we currently use, and this will be mainly based on the cost saved. Only hands-on experience will prove if many current beliefs will hold true.

\section{Disclosure}

The authors report no conflicts of interest in this work.

\section{References}

1. Scheinberg MA, Kay J. The advent of biosimilar therapies in rheumatology - "O brave new world." Nat Rev Rheumatol. 2012;8(7):430-436.

2. Agarwal SK. Core management principles in rheumatoid arthritis to help guide managed care professionals. J Manag Care Pharm. 2011; 17(9 Suppl B):S3-S8.

3. Lapadula G, Ferraccioli GF. Biosimilars in rheumatology: pharmacological and pharmacoeconomic issues. Clin Exp Rheumatol. 2012;30(4 Suppl 73): S102-S106.

4. Carey K. Biosimilars encircle Rituxan, US debates innovator exclusivity. Nat Biotechnol. 2011;29(3):177-178. 
5. Dörner T, Strand V, Castañeda-Hernández G, et al. The role of biosimilars in the treatment of rheumatic diseases. Ann Rheum Dis. 2013;72(3):322-328.

6. Huggett B, Hodgson J, Lähteenmäki R. Public biotech 2009 - the numbers. Nat Biotechnol. 2010;28(8):793-799.

7. Kirchhoff T, Ruof J, Mittendorf T, et al. Cost of illness in rheumatoid arthritis in Germany in 1997-1998 and 2002: cost drivers and cost savings. Rheumatology (Oxford). 2011;50(4):756-761.

8. Dranitsaris G, Amir E, Dorward K. Biosimilars of biological drug therapies: regulatory, clinical and commercial considerations. Drugs. 2011;71(12):1527-1536.

9. [No authors listed]. Approval of biosimilars in the USA - dead ringers? Lancet. 2012;379(9817):686.

10. Colbert RA, Cronstein BN. Biosimilars: the debate continues. Arthritis Rheum. 2011;63(10):2848-2850.

11. Kozlowski S, Woodcock J, Midthun K, Sherman RB. Developing the nation's biosimilars program. $N$ Engl J Med. 2011;365(5):385-388.

12. Azevedo VF, Felippe LR, Machado DM. Opinion of some Brazilian rheumatologists about biosimilars. Rev Bras Reumatol. 2011;51(6): 667-671.

13. Weise M, Bielsky MC, De Smet K, et al. Biosimilars - why terminology matters. Nat Biotechnol. 2011;29(8):690-693.

14. US Food and Drug Administration. Quality Considerations in Demonstrating Biosimilarity to a Reference Protein Product. Rockville (MD): FDA; 2012. Available from: http://www.fda.gov/downloads/ Drugs/GuidanceComplianceRegulatoryInformation/Guidances/ UCM291134.pdf. Accessed June 12, 2013.

15. Jelkmann W. Biosimilar epoetins and other "follow-on" biologics: update on the European experiences. Am J Hematol. 2010;85(10):771-780.

16. Kay J. Biosimilars: a regulatory perspective from America. Arthritis Res Ther. 2011;13(3):112.

17. European Medicines Agency. Guideline on similar biological medicinal products containing biotechnology-derived proteins as active substance: quality issues. 2006. Available from: http://www.ema europa.eu/docs/en_GB/document_library/Scientific_guideline/2009/09/ WC500003953.pdf. Accessed April 19, 2013.

18. Revers L, Furczon E. An introduction to biologics and biosimilars. Part I: Biologics: what are they and where do they come from? Can Pharmacists J. 2010;143(3):134-139.

19. Schellekens H. Biosimilar therapeutics-what do we need to consider? NDT Plus. 2009;2 Suppl 1:i27-i36.

20. Russell AS, Ahluwalla V, Barnabe C, et al. Subsequent entry biologics/ biosimilars: a viewpoint from Canada. Clin Rheumatol. 2012;31(9): 1289-1292.

21. Schneider CK. Biosimilars in rheumatology: the wind of change. Ann Rheum Dis. 2013;72(3):315-318.
22. Schiestl M, Stangler T, Torella C, Cepeljnik, T, Toll H, Grau R. Acceptable changes in quality attributes of glycosylated biopharmaceuticals. Nat Biotechnol. 2011;29(4):310-312.

23. McCamish M, Woollett G. The state of the art in the development of biosimilars. Clin Pharmacol Ther. 2012;91(3):405-417.

24. US Food and Drug Administration. FDA issues draft guidance on biosimilar product development [press release]. Rockville (MD): FDA; February 9, 2012. Available from: http://www.fda.gov/NewsEvents/ Newsroom/PressAnnouncements/ucm291232.htm. Accessed April 19, 2013.

25. European Medicines Agency. Guideline on similar biological medicinal products containing monoclonal antibodies - non-clinical and clinical issues. 2012. Available from: http://www.ema.europa.eu/docs/en_GB/ document_library/Scientific_guideline/2012/06/WC500128686.pdf. Accessed April 19, 2013.

26. Mysler E, Scheinberg M. Biosimilars in rheumatology: a view from Latin America. Clin Rheumatol. 2012;31(9):1279-1280.

27. US Food and Drug Administration. Biosimilars: questions and answers regarding implementation of the Biologics Price Competition and Innovation Act of 2009. 2012. Available from: http://www.fda. gov/downloads/Drugs/GuidanceComplianceRegulatoryInformation/ Guidances/UCM273001.pdf. Accessed April 19, 2013.

28. Braido F, Holgate S, Canonica GW. From "blockbusters" to "biosimilars": an opportunity for patients, medical specialists and health care providers. Pulm Pharmacol Ther. 2012;25(6):483-486.

29. Celltrion Healthcare. CT-P13 clinical trial. Available from: http://www. celltrionhealthcare.com/products/ct-p13/patient/product/product.asp. Accessed April 4, 2013.

30. Park W, Hrycaj P, Kovalenko V, et al. A randomized, double-blind, phase 1 study demonstrates equivalence in pharmacokinetics safety, and efficacy of CT-P13 and Infliximab in patients with ankylosing spondylitis [abstract]. Ann Rheum Dis. 2012;71 Suppl 3:111.

31. Yoo D, Miranda P, Piotrowski M, et al. A randomized, double-blind, phase 3 study demonstrates clinical equivalence of CT-P13 to infliximab when co-administered with methotrexate in patients with active rheumatoid arthritis [abstract]. Ann Rheum Dis. 2012;71 Suppl 3:359.

32. [No authors listed]. Celltrion's first biosimilar antibody is about to reach the market. Biosimilar News. 2012. Available from: http://www. biosimilarnews.com/celltrions-first-biosimilar-antibody-is-about-toreach-the-market. Accessed April 4, 2013.

33. Burgos-Vargas R, Catoggio LJ, Galarza-Maldonado C, Ostojich K, Cardiel MH. Current therapies in rheumatoid arthritis: a Latin American perspective. Reumatol Clin. 2013;9(2):106-112.

34. Nature Publishing Group. Similarity trials [editorial]. Nat Biotechnol. 2011;29(1):1.
Biosimilars

\section{Publish your work in this journal}

Biosimilars is an international, peer-reviewed, open access journal focusing on the manufacture, development and medicinal use of biopharmaceutical compounds considered similar to an innovator agent. Specific topics covered in the journal include: Regulatory issues and pathways; manufacturing processes; chemical composition and

\section{Dovepress}

structure; quality and purity; patent issues; bioequivalence and interchangeability; clinical efficacy data; patient perspectives. The manuscript management system is completely online and includes a very quick and fair peer-review system. Visit http://www.dovepress.com/ testimonials.php to read real quotes from published authors. 